\title{
Evaluation of an antibiogram-resistogram typing scheme for methicillin-resistant Staphylococcus aureus
}

\author{
A. S. ROSSNEY, D. C. COLEMAN* and C. T. KEANE
}

Department of Clinical Microbiology, Sir Patrick Dun Research Laboratory, Trinity College Dublin, Central Pathology Laboratory, St James's Hospital, James's Street, Dublin 8 and * Department of Microbiology, Trinity College, Dublin, Ireland

\begin{abstract}
Summary. Between Dec. 1992 and Aug. 1993, the MRSA population in the Federated Dublin Voluntary Hospitals and St James's Hospital group was studied with an antibiogramresistogram (AR) typing scheme in which AR patterns were determined by testing susceptibility to 22 antibiotics and chemicals by a modified Stokes' disk diffusion technique. The typing scheme divided this MRSA population into 31 AR types but $90 \%$ of isolates belonged to seven types. Isolates belonging to the most frequently occurring types (AR types 13 and 14) differed only in their reaction to lincomycin (or clindamycin) and could not be distinguished by phage typing, plasmid profiling or restriction endonuclease analysis. The AR typing scheme showed that the incidence of different AR types varied in different hospitals and changed during the study period. This typing method differentiated a strain of MRSA responsible for a nosocomial outbreak in an intensive care unit from other MRSA isolated in the unit, and has distinguished imported strains from local ones. In one hospital, AR typing showed that, although a major outbreak occurred with one AR type, there was also a series of smaller outbreaks with other AR types. The technique can be performed in the diagnostic laboratory and results were available within $24 \mathrm{~h}$.
\end{abstract}

\section{Introduction}

Methicillin-resistant Staphylococcus aureus (MRSA) is an important nosocomial pathogen with an increasing incidence worldwide in recent years. ${ }^{1-4}$ In Ireland MRSA became a major problem in the late $1970 \mathrm{~s}^{5,6}$ and the incidence is now increasing. Since 1987 this increase has become evident in the group of hospitals served by this laboratory. ${ }^{\text {? }}$

An antibiogram-resistogram (AR) typing scheme able to provide results within $24 \mathrm{~h}$ and suitable for use in the diagnostic laboratory is described in an accompanying paper. ${ }^{8}$ Here we report an evaluation of this typing scheme.

\section{Materials and methods}

\section{Hospitals}

The Microbiology Department of the Central Pathology Laboratory at St James's Hospital (SJH) serves SJH and the Federated Dublin Voluntary Hospitals (FDVH) group of hospitals. This group consists of three acute general hospitals and one paediatric hospital. In 1992, the hospital group had a bed complement of 1243 and 56330 admissions, with an MRSA incidence of $0.7 \%$ of admissions.

\section{Antibiogram-resistogram typing}

Bacterial isolates. MRSA isolated between 20 Dec. 1992 and 31 Aug. 1993 were typed. One isolate from each patient was tested unless routine susceptibility test results indicated the presence of more than one strain. Isolates (535) were collected from 347 patients, 12 staff members and four environmental specimens. A further 26 isolates from six other Dublin hospitals were also typed. Fresh isolates were typed as soon as possible after isolation or stored frozen at $-70^{\circ} \mathrm{C}$ on cryoprotective beads (Protect; Technical Service Consultants Ltd).

Isolates were identified by testing for the production of clumping factor, coagulase and thermostable DNAse. ${ }^{9,10}$ Since 5 April 1993, isolates were also identified with a $S$. aureus identification kit (Pastorex Staph-Plus; Sanofi Diagnostics Pasteur). Methicillin susceptibility was tested at $30^{\circ} \mathrm{C}$ on Columbia blood agar (CBA) with $25-\mu \mathrm{g}$ methicillin strips (Mast) and a 


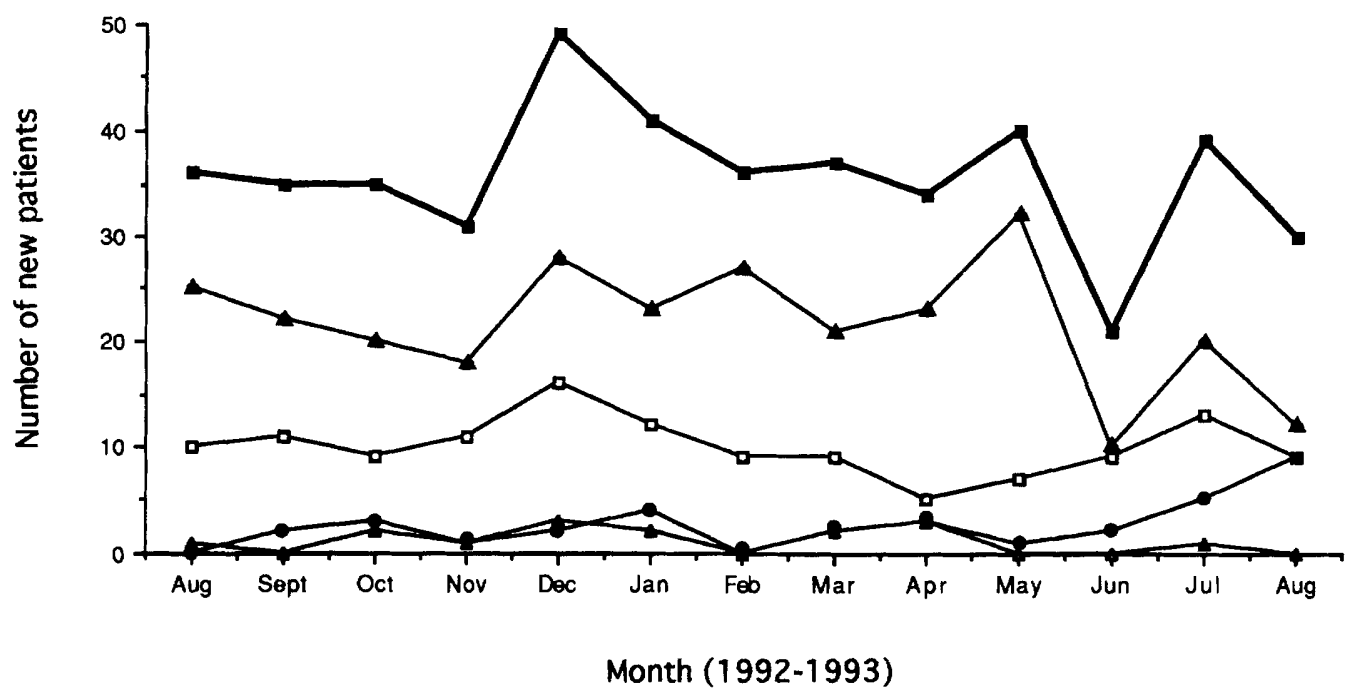

Fig. 1. Incidence of MRSA isolated in the Federated Dublin Voluntary Hospitals and St James's Hospital from Aug. 1992 to Aug. 1993 (new MRSA patients only included); -- , hospital $1 ;-\square-$, hospital $2 ;-\mathbf{\Delta}-$, hospital $3 ;-\triangle-$, hospital $4 ;-\square-$, total.

Table I. Most frequently encountered AR patterns

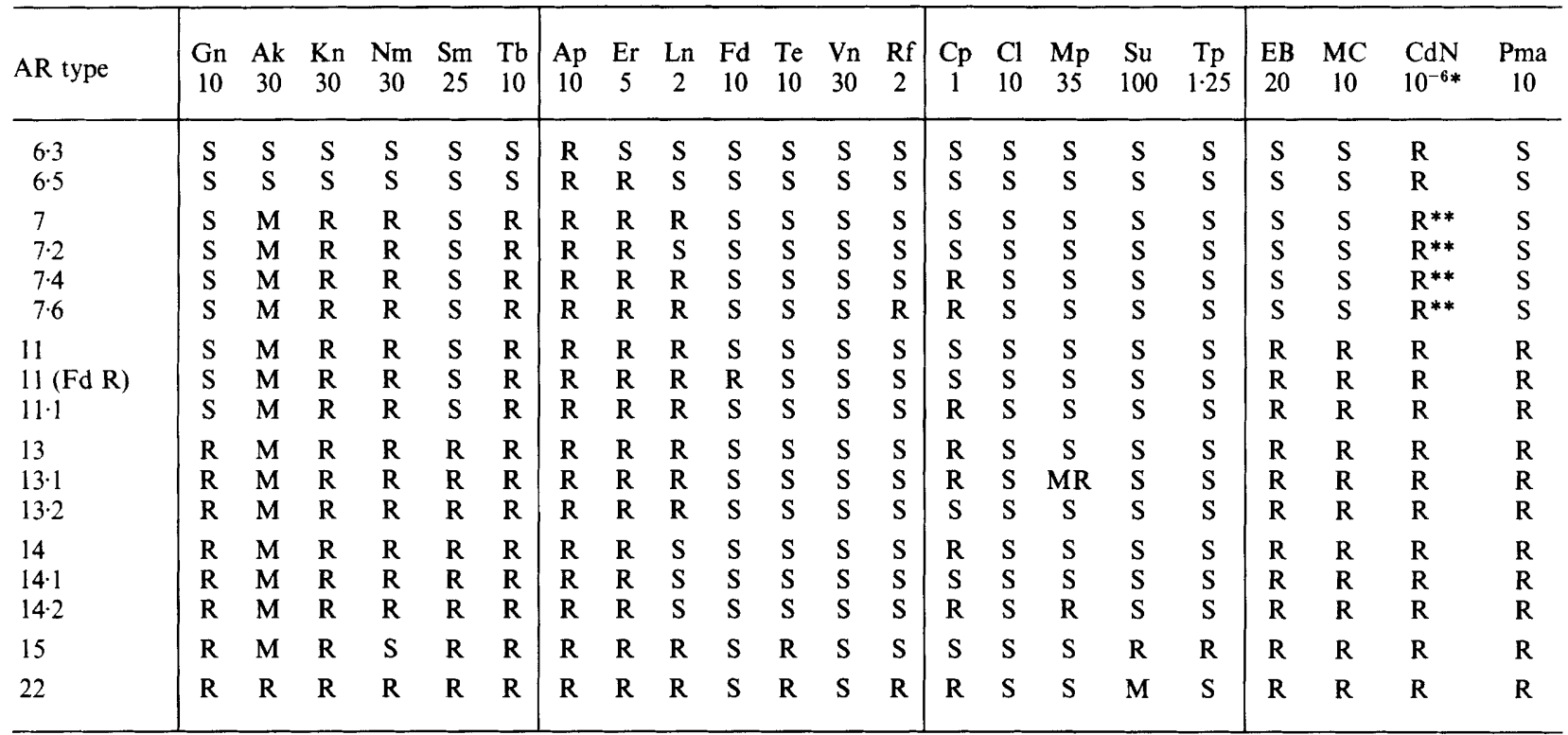

$\mathrm{R}$ resistant, $\mathrm{S}$ sensitive, $\mathrm{M}$ moderate, for other abbreviations see Materials and methods.

${ }^{*}$ Cadmium nitrate concentration $10^{-6}$ mols. ${ }^{* *}$ May be sensitive to higher concentration $(1 \mathrm{~mm})$ cadmium disks. ${ }^{8}$

Table II. AR type distribution among MRSA isolates (one isolate per AR type from each patient) from patients in $\mathrm{St}$ James Hospital and the Federated Dublin Voluntary Hospitals (Jan.-Aug. 1993)

\begin{tabular}{|c|c|c|c|c|c|c|c|c|}
\hline \multirow{2}{*}{ AR type } & \multicolumn{8}{|c|}{ Number of isolates } \\
\hline & Jan. & Feb. & Mar. & April & May & June & July & Aug. \\
\hline 6 & 1 & 1 & 2 & 6 & 10 & 5 & 5 & 6 \\
\hline 7 & 9 & 13 & 14 & 4 & 12 & 9 & 8 & 7 \\
\hline 11 & 17 & 15 & 11 & 11 & 7 & 5 & 11 & 9 \\
\hline 13 & 23 & 18 & 19 & 19 & 21 & 15 & 19 & 20 \\
\hline 14 & 12 & 19 & 19 & 24 & 27 & 22 & 17 & 16 \\
\hline 15 & 7 & 3 & 0 & 1 & 1 & 0 & 0 & 0 \\
\hline 22 & 2 & 3 & 5 & 8 & 3 & 2 & 0 & 0 \\
\hline
\end{tabular}

heavy inoculum. ${ }^{11,12}$ The methicillin-resistant $S$. aureus NCTC 10442 was included as a positive control. ${ }^{13}$
Susceptibility testing. Susceptibility testing was performed by a modified Stokes' disk diffusion technique in which $S$. aureus ATCC 25923 replaced S. aureus NCTC 6571 as the control organism. ${ }^{8}$ Isolates were tested on Diagnostic Sensitivity Test Agar DST, Oxoid) against the panel of antimicrobial agents listed below. Commercially-available antibiotic disks were purchased from Oxoid and the sources of other antimicrobial agents are specified. The following antimicrobial agents (concentration/disk) were tested: amikacin $30 \mu \mathrm{g}$ (Ak), ampicillin $10 \mu \mathrm{g}$ (Ap), cadmium nitrate $10^{-6} \mathrm{mols}$ (Cdn, Aldrich), chloramphenicol $10 \mu \mathrm{g}(\mathrm{Cl})$, ciprofloxacin $1 \mu \mathrm{g}(\mathrm{Cp})$, erythromycin $5 \mu \mathrm{g}$ (Er), ethidium bromide $20 \mu \mathrm{g}$ (EB, Sigma), fusidic acid $10 \mu \mathrm{g}(\mathrm{Fd})$, gentamicin $10 \mu \mathrm{g}(\mathrm{Gn})$, kanamycin $30 \mu \mathrm{g}$ $(\mathrm{Kn})$, lincomycin $2 \mu \mathrm{g}$ (Ln), mercuric chloride $10 \mu \mathrm{g}$ (Mc, BDH), mupirocin $35 \mu \mathrm{g}(\mathrm{Mp})$, neomycin $30 \mu \mathrm{g}$ $(\mathrm{Nm})$, phenyl mercuric acetate $10 \mu \mathrm{g}$ (Pma, Sigma), 
Hospital 1
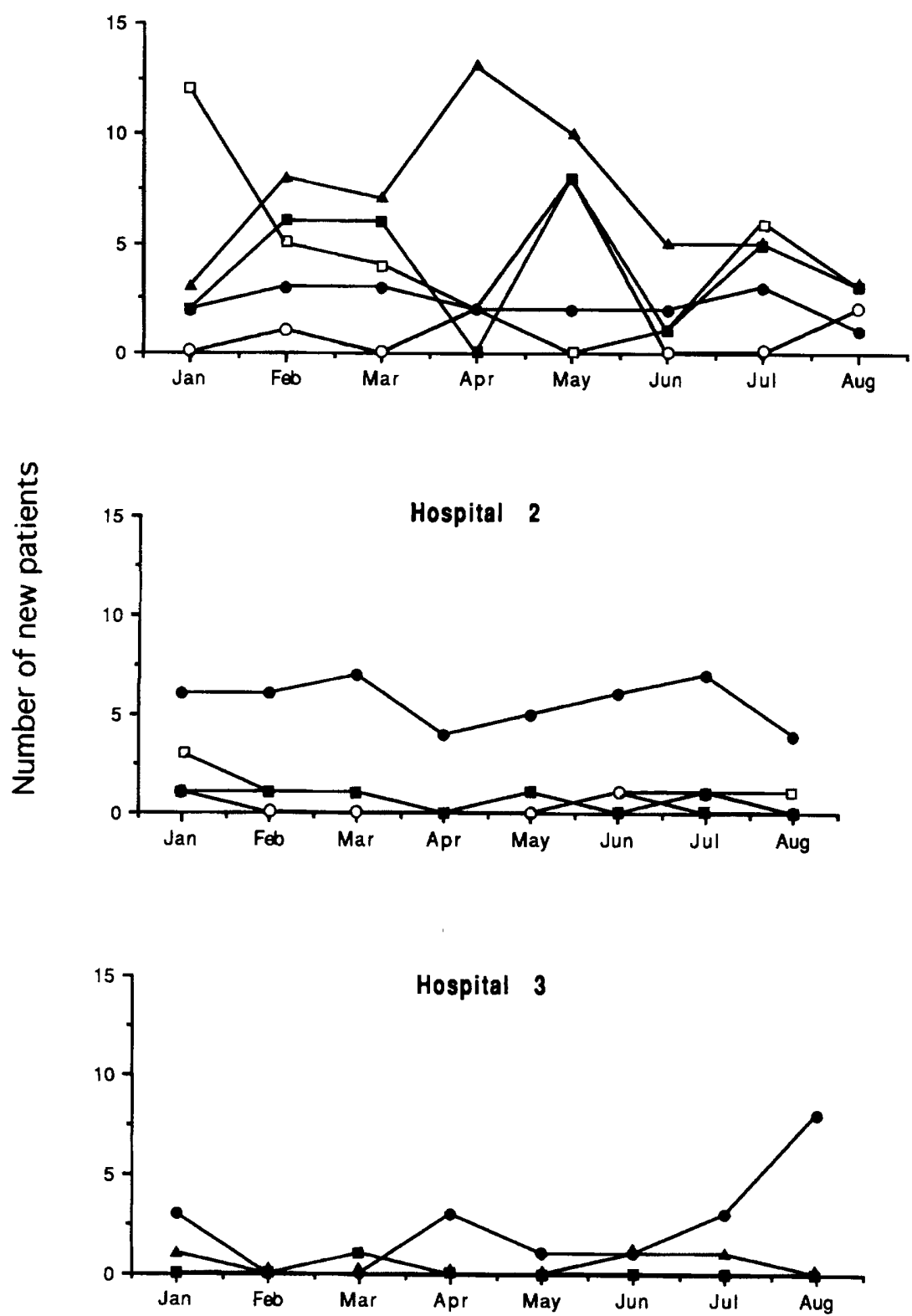

Month (1993)

Fig. 2. Distribution of the five most frequently occurring AR types among MRSA isolates in the Federated Dublin Voluntary Hospitals and St James's Hospital (hospitals 1, 2 and 3) from Jan. to Aug. 1993 (new MRSA patients only included); -O-, AR 6; -,-- AR $7 ;-\square-$, AR $11 ;-\mathbf{-}_{-}^{-}$AR $13 ;-\boldsymbol{\Delta}-$, AR 14.

rifampicin $2 \mu \mathrm{g}(\mathrm{Rf})$, streptomycin $25 \mu \mathrm{g}(\mathrm{Sm})$, sulphonamide $100 \mu \mathrm{g}(\mathrm{Su})$, tetracycline $10 \mu \mathrm{g}(\mathrm{Te})$, tobramycin $10 \mu \mathrm{g}(\mathrm{Tb})$, trimethoprim $1 \cdot 25 \mu \mathrm{g}(\mathrm{Tp})$ and vancomycin $30 \mu \mathrm{g}(\mathrm{Vn})$. Safety precautions were observed when handling and disposing of ethidium bromide, cadmium nitrate, mercuric chloride and phenyl mercuric acetate.

Care was taken to ensure that the inoculum density produced the required "dense but not confluent" growth and that incubation was continued for a minimum 18-h period. Zones smaller than the control were measured with a pair of calipers and the zone sizes were recorded. Susceptibility category was interpreted according to the Stokes' technique criteria.

\section{Typing nomenclature}

Isolates were assigned an AR type number on the basis of the susceptibility pattern produced as described previously. ${ }^{8}$

\section{Results}

The incidence of MRSA in this group of hospitals between Aug. 1992 and Aug. 1993 is shown in fig. 1. AR typing divided this MRSA population into $31 \mathrm{AR}$ types (seven types were further divided into subtypes); $90 \%$ of all isolates belonged to seven types (table I). The relative frequency with which these types occurred 
AR6
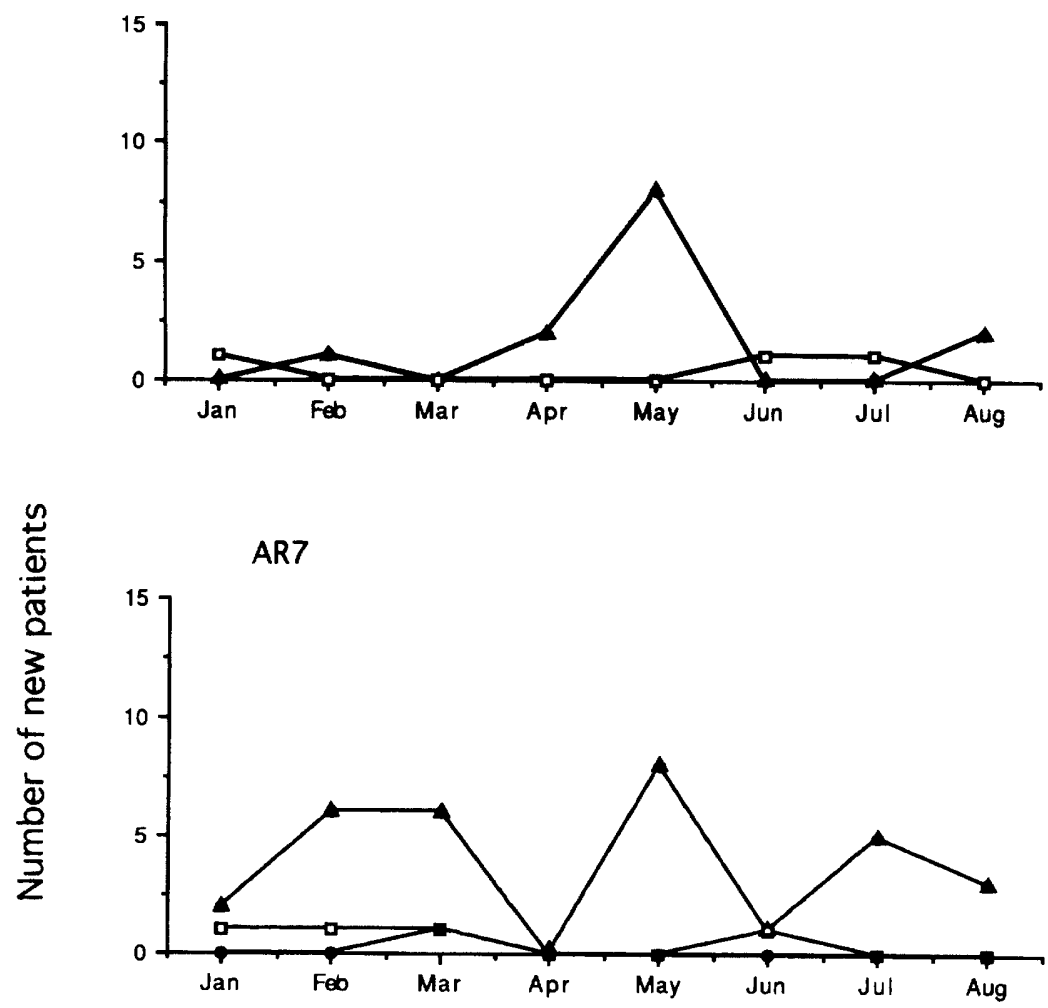

AR11

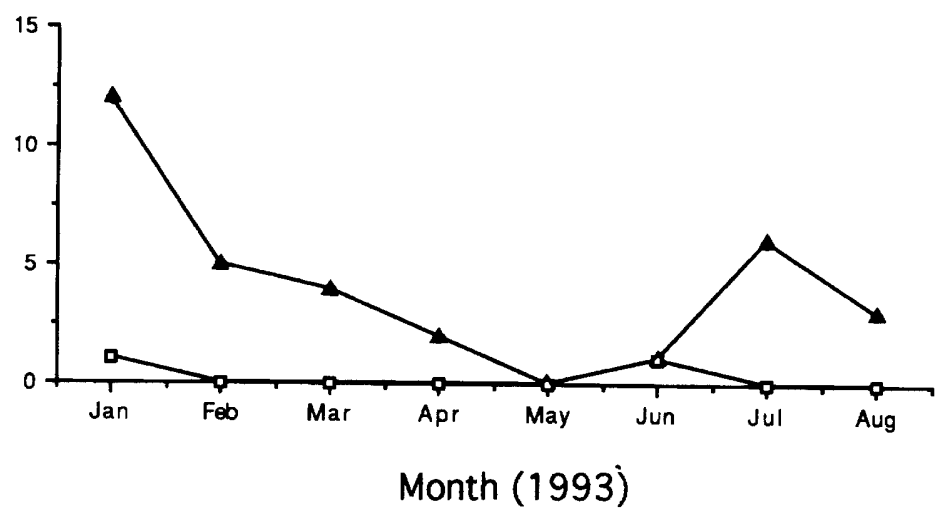

Fig. 3. Distribution of antibiogram/resistogram (AR) types AR 6, 7 and 11 among MRSA isolates from new MRSA patients in the Federated Dublin Voluntary Hospitals and St James's Hospital (Jan.-Aug. 1993); $\mathbf{\Delta -}$, hospital $1 ;-\square-$, hospital 2 ; -, hospital 3.

among isolates in this hospital group is shown in table II.

Fig. 2 shows the distribution of the five most common AR types among patients acquiring MRSA in hospitals 1, 2 and 3 respectively. Because there was a low incidence of MRSA in hospital 4, the AR type distribution for this hospital is not shown. The relative proportion of patients yielding AR types 13 and 14 in hospitals 1 and 2 was markedly different $(8.9 \%$ and $29.7 \%$ in hospital 1 compared with $60 \%$ and $12.9 \%$ in hospital 2). In hospital 3, the incidence of AR type 13 was rising.

The higher incidence (fig. 1) and more complex AR type distribution pattern (fig. 2) among MRSA isolates from hospital 1 was due in part to the fact that hospital 1 was the largest hospital in the group (in 1992, hospitals 1,2, 3 and 4 had 722, 250,180 and 91 beds respectively). In hospital 1 there was a sharp increase in AR type 14 between January and April and a decrease in AR type 11 during the same period (fig. 2).

The remaining two frequently occurring AR types in hospital 1 were AR types 15 and 22 . With one exception, all isolates of AR type 15 were cultured from patients admitted to the burns unit, where in January 1993 isolates from $60 \%$ of MRSA patients belonged to AR type 15. Although prevalent during the first 2 months of 1993, isolates of AR type 15 have not been isolated from new MRSA patients since Feb. 1993. AR type 22 caused an outbreak of infection in the intensive care unit (ICU) between January and June, 1993.

When the incidence of each of the more commonly occurring AR types was analysed (figs. 3 and 4), it could be seen that in addition to a major outbreak with 


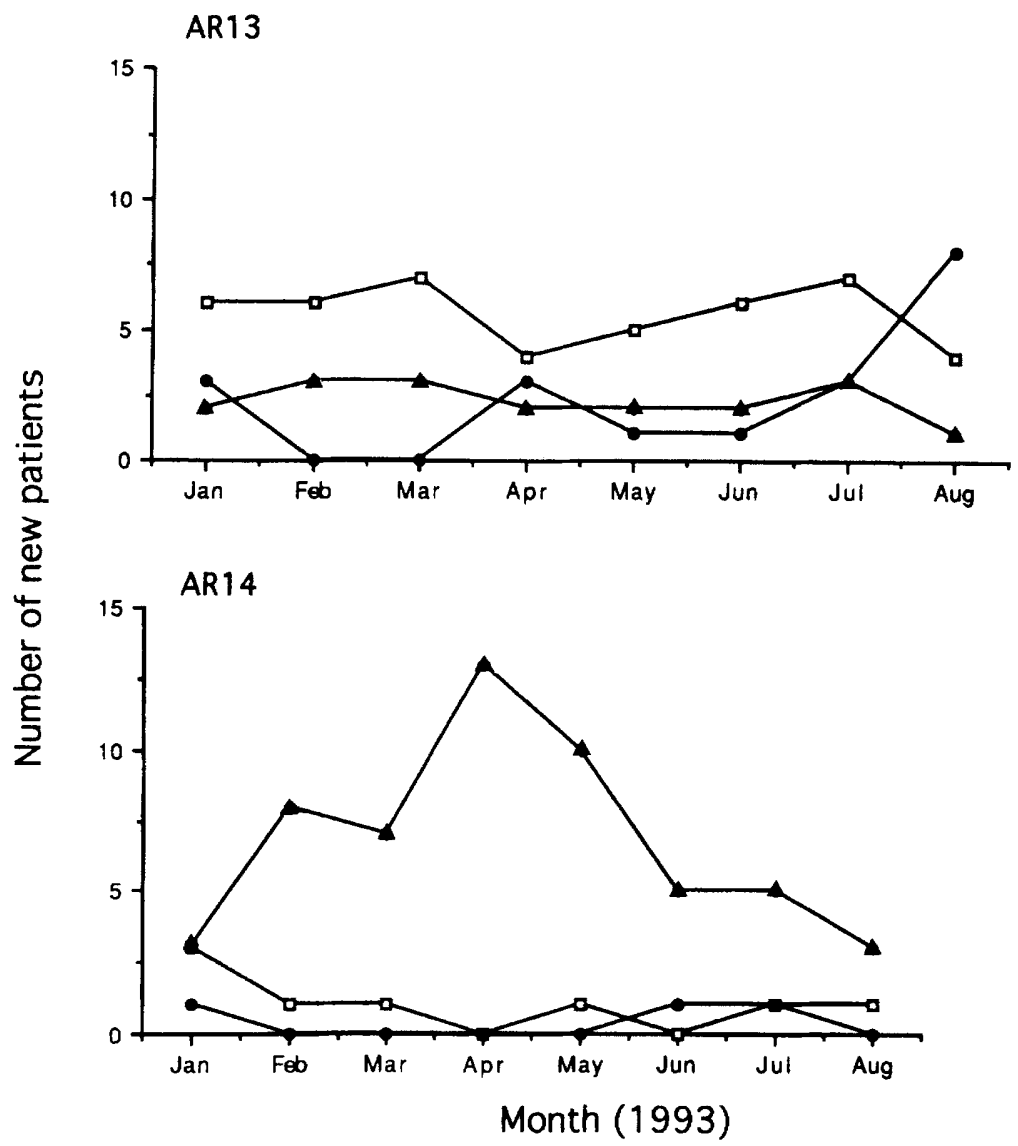

Fig. 4. Distribution of antibiogram/resistogram (AR) types AR 13 and 14 among MRSA isolates from new MRSA patients in the Federated

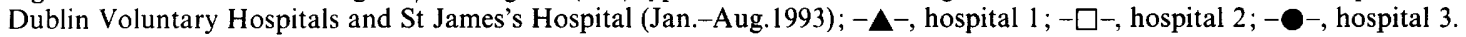

AR type 14 in hospital 1 and AR type 13 in hospital 2, a series of smaller outbreaks was also occurring. For example, in hospital 1 between April and June, there was a peak in the incidence of AR type 6, while AR type 7 showed a series of peaks between January and August. In contrast, the incidence of AR type 11 fell steadily until May but increased between June and July.

In addition to the more frequently occurring types, recognition of new AR types may confirm the introduction of new strains; for example, AR type 23 was isolated from a patient who had been hospitalised in Croatia. ${ }^{14}$ AR typing detected an unusual rifampicin-resistant strain (AR type 22) that caused the nosocomial ICU outbreak mentioned earlier. The typing scheme differentiated the outbreak strain from other MRSA isolates cultured from patients and staff in the ICU during the outbreak.

The emergence of mupirocin resistance was observed in MRSA in this hospital group in 1993; $82 \%$ of mupirocin-resistant isolates belonged to AR type 13 and most $(82 \%)$ of these isolates were cultured from patients in hospital 2. Patients in other hospitals from whom this mupirocin-resistant MRSA was isolated were frequently found to have been transferred from hospital 2.

AR types 13 and 14 accounted for half $(50.8 \%)$ the MRSA isolates typed. These types differed only with respect to lincomycin (or clindamycin) resistance and while it would be preferable not to rely on a single reaction to distinguish two types, epidemiological evidence from this study supported the conclusion that AR types 13 and 14 were different.

When AR typing was applied to 26 isolates from six other Dublin hospitals, all isolates belonged to AR types encountered in the SJH/FDVH hospital group; $56 \%$ of isolates belonged to AR type 13 (compared with $60 \%$ in hospital 2).

\section{Discussion}

Although MRSA have been a major problem in Dublin for $>15$ years, until recently only a limited number of MRSA phenotypes had been described. ${ }^{15,16}$ The plasmid profiles and AR types of the majority of the MRSA population in this study differed from earlier phenotypes and showed sufficient diversity to make AR typing feasible. In the ideal typing scheme, not more than $5 \%$ of epidemiologically unrelated isolates should belong to the same type. ${ }^{17}$ In practice, it may be difficult to determine whether isolates are epidemiologically unrelated in a city like Dublin, where many medical specialities are centralised and there is constant patient and staff transfer among hospitals.

Approximately $60 \%$ of MRSA throughout the city appear to belong to AR type 13 but many of these isolates may be epidemiologically related. This result is based on a small random sample and may be an indication of the extent of inter-hospital MRSA cross- 
infection in Dublin. A prospective citywide survey would be helpful to determine the extent of the problem and the AR type distribution of the overall population.

Within this hospital group, AR typing has shown an obvious trend in hospitals 2 and 3 where AR type 13 isolates predominated. In hospital 1 a more complex pattern has emerged. Although the incidence of all MRSA isolates rose in April (fig. 1), AR type analysis indicated that the incidence of AR type 14 increased in March and its decline in April coincided with an increasing incidence of AR types 6 and 7 (fig. 2). Monitoring the varying incidence of different AR types can provide an early indication of trends that may be difficult to perceive in areas with an extensive endemic MRSA problem. AR typing may also detect small incidences of cross-infection that might otherwise go unnoticed.

The sharp increase in MRSA incidence in hospital 1 in April may have been due to an intensive screening programme and the introduction of salt enrichment to improve MRSA isolation from screening specimens. ${ }^{18}$ These measures were introduced in response to an outbreak in the ICU. The decreasing incidence since May may be a consequence of these measures and the introduction of stringent infection control protocols. Between April and June, the incidence of AR type 14 decreased dramatically.

The most common AR types encountered, AR types 13 and 14, appear to be closely related. With AR typing, the only distinguishing reaction occurred with lincomycin (or clindamycin). AR types 13 and 14 are indistinguishable on phage typing, plasmid profiling, restriction endonuclease analysis or enterotoxin production. Epidemiological evidence indicates that they are two distinct types as shown by the differing incidence in hospitals 1 and 2 (fig. 2) and by the fact that when MRSA of AR type 13 was isolated in hospital 1, the patient had often been transferred from hospital 2. Further evidence may be obtained from hospital 2 where mupirocin resistance emerged in AR type 13. The initial mupirocin-resistant isolates were cultured from patients who had been on prolonged mupirocin therapy. Resistance was almost entirely restricted to AR type 13 and although this was the predominant type isolated in this hospital, AR type 14

\section{References}

1. Duckworth GJ. Methicillin-resistant Staphylococcus aureus. In: Reeves DS, Geddes AM (eds) Recent advances in infection number three. Edinburgh, Churchill Livingstone. 1989: 189-207.

2. Cookson BD. Epidemiology and control of nosocomial methicillin-resistant Staphylococcus aureus. Curr Opin Infect Dis 1991 ; 4: 530-535.

3. Cafferkey MT. Therapy for staphylococcal infection. Curr Opin Infect Dis 1991; 4: 757-763.

4. Lyon BR, Skurray R. Antimicrobial resistance of Staphylococcus aureus: genetic basis. Microbiol Rev 1987; 51: 88-134.

5. Cafferkey MT, Coleman D, McGrath et al. Methicillin-resistant Staphylococcus aureus in Dublin 1974-84. Lancet 1985; 2 : 705-708. was also present but mupirocin resistance did not emerge in this strain.

The distinction between AR types 13 and 14 was helpful in hospital 3 where the increasing incidence of MRSA (fig. 2) was thought to have resulted from the admission of an MRSA patient from an external hospital. The majority of MRSA isolates from hospital 3 belonged to AR type 13 but the isolate from the presumed index case was AR type 14. Patients were frequently transferred to this hospital from hospital 2 where AR type 13 predominated. Thus, it was likely that a transferred patient from hospital 2 and not the external hospital was the source. Confirmation of the difference between AR types 13 and 14 may be possible by either pulsed field gel electrophoresis or random amplification of polymorphic DNA by polymerase chain reaction (RAPD-PCR). ${ }^{19-21}$

In addition to epidemiological information, AR typing can provide clinically useful information on the antimicrobial susceptibility pattern of the MRSA population. For example, this study population was largely susceptible to trimethoprim, tetracycline and fusidic acid while the most frequently occurring AR types were ciprofloxacin resistant (table I). Information regarding susceptibility to antibiotics not routinely tested in the diagnostic laboratory may also be available from the AR type: for example, if mupirocin is not routinely tested, the AR type will indicate resistant isolates.

This AR typing method has proved valuable in monitoring the MRSA population in this group of hospitals. It has demonstrated the heterogeneity of the present MRSA population and the extent to which this population is changing, and has recognised the introduction of new strains. AR typing has shown that, in addition to major outbreaks, a series of concurrent small outbreaks contributed to the MRSA problem in this hospital group.The method is suitable for use in the diagnostic laboratory and produces results within $24 \mathrm{~h}$.

We thank Dr R. Marples, Staphylococcal Reference Laboratory, Public Health Laboratory Service, Colindale, London for supplying typing bacteriophages and Harriett Pomeroy, Department of Microbiology, Trinity College Dublin for guidance in phage typing technique. We gratefully acknowledge the gift of fusidic acid from Leo Laboratories Limited and thank Dr F. Falkiner for critically reviewing the manuscript.

6. Morgan MG, Harte-Barry MJ. Methicillin-resistant Staphylococcus aureus: a ten-year survey in a Dublin hospital. $J$ Hosp Infect 1989; 14: 357-362.

7. Rossney AS, Pomeroy HM, Keane CT. Staphylococcus aureus phage typing, antimicrobial susceptibility patterns and patient data correlated using a personal computer: advantages for monitoring the epidemiology of MRSA. $J$ Hosp Infect 1994; (in press).

8. Rossney AS, Coleman DC, Keane CTK. Antibiogramresistogram typing scheme for methicillin-resistant Staphylococcus aureus. J Med Microbiol 1994; 41 : 430-440.

9. Rossney AS, English LF, Keane CT. Coagulase testing compared with commercial kits for routinely identifying Staphylococcus aureus. J Clin Pathol 1990; 43: 246-252.

10. Lachica RVF, Genigeoris C, Hoeprich PD. Metachromatic agar-diffusion methods for detecting staphylococcal nuclease activity. Appl Microbiol 1971; 21 : 585-587. 
11. Annear DI. The effect of temperature on resistance of Staphylococcus aureus to methicillin and some other antibiotics. Med J Aust 1968; i: 444446.

12. Hewitt JH, Coe AW, Parker MT. The detection of methicillin resistance in Staphylococcus aureus. J Med Microbiol 1969; 2: $443-456$.

13. Anon. Antibiotic sensitivity tests: control strains. In Catalogues of the National Collection of Type Cultures. London, Public Health Laboratory Service. 1989: 127.

14. Rossney AS, McCarthy EM, Coleman DC. Rapid recognition of a new MRSA strain in Dublin. Irish J Med Sci 1993; 162 Suppl 11:26.

15. Coleman DC, Pomeroy H, Estridge JK et al. Susceptibility to antimicrobial agents and analysis of plasmids in gentamicin- and methicillin-resistant Staphylococcus aureus from Dublin hospitals. J Med Microbiol 1985; 20 $157-167$.

16. Carroll JD, Pomeroy HM, Russell RJ et al. A new methicillinand gentamicin-resistant Staphylococcus aureus in Dublin: molecular genetic analysis. $J$ Med Microbiol 1989; 28: 15-23.

17. Marples RR. Epidemiologic typing of coagulase-negative staphylococci. Curr Opin Infect Dis 1992; 5: 530-535.

18. Prevost G, Pottecher B, Dahlet M, Bientz M, Mantz JM, Piemont Y. Pulsed field gel electrophoresis as a new epidemiological tool for monitoring methicillin-resistant Staphylococcus aureus in an intensive care unit. $J$ Hosp Infect 1991; 17: 255-269.

19. Saulnier P, Bourneix C, Prevost G, Andremont A. Random amplified polymorphic DNA assay is less discriminant than pulsed-field gel electrophoresis for typing strains of methicillin-resistant Staphylococcus aureus. J Clin Microbiol 1993; 31 : 982-985.

20. Van Belkum A, Bax R, Peerbooms P, Goessens WHF, Van Leeuwen N, Quint WGV. Comparison of phage typing and DNA fingerprinting by polymerase chain reaction for discrimination of methicillin-resistant Staphylococcus aureus strains. J Clin Microbiol 1993; 31: 798-803. 